\title{
Pharmacotherapy for treatment-resistant schizophrenia
}

This article was published in the following Dove Press journal:

Neuropsychiatric Disease and Treatment

16 March 201I

Number of times this article has been viewed

\author{
Meghan E Mcilwain ${ }^{1,2}$ \\ Jeff Harrison' \\ Amanda J Wheeler ${ }^{1,3}$ \\ Bruce R Russell, \\ 'School of Pharmacy, Faculty \\ of Medical and Health Sciences, \\ University of Auckland, Auckland, \\ New Zealand; ${ }^{2}$ Centre for Brain \\ Research, Faculty of Medical and \\ Health Sciences, University of \\ Auckland, Auckland, New Zealand; \\ ${ }^{3}$ School of Human Services, Griffith \\ University, Queensland, Australia
}

Correspondence: Bruce Russell School of Pharmacy, Faculty of Medical and Health Sciences, University of Auckland, Private Bag 92019, Auckland, New Zealand

Tel +6499236429

Fax +649376 7192

Email b.russell@auckland.ac.nz

\begin{abstract}
Schizophrenia is a disabling mental illness with a lifetime prevalence of $0.7 \%$ worldwide and significant, often devastating, consequences on social and occupational functioning. A range of antipsychotic medications are available; however, suboptimal therapeutic response in terms of psychotic symptoms is common and affects up to one-third of people with schizophrenia. Negative symptoms are generally less amenable to treatment. Because of the consequences of inadequate symptom control, effective treatment strategies are required for people with treatment-resistant schizophrenia. Clozapine has been shown to be more effective than other antipsychotics in treatment-resistant populations in several studies; however, the occurrence of adverse effects, some of which are potentially life-threatening, are important limitations. In addition to those who are intolerant to clozapine, only $30 \%$ to $50 \%$ experience clinically significant symptom improvement. This review describes the recent evidence for treatment strategies for people not responding to nonclozapine antipsychotic agents and people not responding or only partially responding to clozapine.
\end{abstract}

Keywords: antipsychotic, refractory, clozapine

\section{Introduction}

Schizophrenia is a disabling mental illness with a lifetime prevalence of $0.7 \%$ worldwide. ${ }^{1}$ Typically beginning in early adolescence, outcomes for patients are variable but the course of illness is chronic, often marked with periods of relapse despite treatment. Schizophrenia has a significant and often devastating impact on social and occupational functioning for patients, often due to residual negative symptoms and cognitive deficits. ${ }^{2}$ This may manifest as the decreased likelihood of living independently, being in an intimate relationship, achieving formal education, or being in paid employment. ${ }^{3-6}$ A range of antipsychotic medications is available, including first-generation antipsychotics (FGAs) and second-generation antipsychotics (SGAs). ${ }^{7,8}$ However, suboptimal therapeutic response in terms of psychotic symptoms is common and affects up to one-third of people. ${ }^{9}$ Negative symptoms may be classified as primary (part of the disease process itself) or secondary (to factors such as depression, drug-induced akinesia, or a suspicious withdrawal) ${ }^{10}$ and are generally less amenable to treatment. ${ }^{11,12}$ Antipsychotic agents have no demonstrable efficacy for primary enduring or "deficit" negative symptoms. ${ }^{13}$ Improvements in this symptom domain are largely a consequence of a reduction in positive symptoms. ${ }^{14,15}$ While antipsychotic agents improve attention in people with schizophrenia, ${ }^{16,17}$ the effects observed for other cognitive impairments are inconsistent $^{18}$ and may include worsening. ${ }^{19,20}$ The net impact of an antipsychotic agent 
on cognitive function is determined by the beneficial effect on attention and adverse effects related to anticholinergic activity and extrapyramidal side effects (EPSE). ${ }^{21}$ Furthermore, it has been postulated that a practice effect may account for beneficial effects observed. ${ }^{22}$ There are no apparent consistent differences between antipsychotic agents with respect to their effect on cognition. ${ }^{23-25}$ Because of the consequences of inadequate symptom control, effective treatment strategies are required for people with treatment-resistant schizophrenia (TRS).

Several definitions of treatment-resistant schizophrenia exist and vary in their specificity. The criteria employed by Kane et al to define treatment-resistant (or treatmentrefractory) schizophrenia in the pivotal trial comparing clozapine to chlorpromazine is used frequently in clinical trials and audit settings. ${ }^{26,27}$ Kane et al classified participants as treatment-resistant if: improvement had not been demonstrated after 3 periods of treatment with antipsychotics (from 2 or more different chemical classes) in the previous 5 years equivalent to $1000 \mathrm{mg}$ /day of chlorpromazine (CPZ) for 6 weeks and participants had had no episodes of good functioning in the previous 5 years, Brief Psychiatric Rating Scale (BPRS) total score $\geq 45$, Clinical Global Impressions (CGI) score $\geq 4$, and score $\geq 4$ on 2 or 4 positive symptoms items. ${ }^{26}$ Conley and Kelly presented a modified version of these criteria to reflect clinical practice patterns and a better understanding of optimal dosing: 2 antipsychotic trials (400-600 mg CPZ equivalents per day) for 4 to 6 weeks with no clinical improvement, no period of good social or occupational functioning for $>5$ years, BPRS total score $>45$, and a score of $>4$ on 2 of 4 positive items. ${ }^{28}$

Clozapine has been shown to be more effective than other antipsychotics in treatment-resistant populations in several studies; however, the occurrence of adverse effects, some of which are potentially life-threatening, are important limitations. In addition to those who are intolerant to clozapine, only $30 \%$ to $50 \%$ experience clinically significant symptom improvement. ${ }^{29,30}$ This has prompted unlicensed prescribing and antipsychotic combination strategies (with or without clozapine) for which there is the potential for increased side effects and little robust evidence to support this practice.

This review will summarize key studies and recent evidence for treatment strategies for people not responding to nonclozapine antipsychotic agents and people not responding or only partially responding to clozapine. The literature reviewed was identified by a systematic search of Ovid Medline \& Medline In-Process, Embase (combined file 1947 to present), Cochrane Central Register of Controlled
Trials (CENTRAL/CCTR), and PsycINFO, supplemented by hand searches of reference lists. The evidence is presented in 3 sections: clozapine monotherapy versus other antipsychotics, clozapine augmentation strategies, and options for clozapine-intolerant or clozapine-resistant people. The first section is divided into 2 parts comparing clozapine monotherapy to FGAs and SGAs; each part is stratified by the level of evidence presented. The clozapine augmentation section is first stratified by level of evidence (meta-analysis or randomized controlled trial) then by specific treatment strategy. The structure of this section reflects the relative availablity of evidence for the treatment combinations considered. The third section, treatment options for those who are intolerant or resistant to clozapine, discusses alternative antipsychotic monotherapy and nonpharmacological treatments.

\section{Clozapine monotherapy}

The World Psychiatric Association Section on Pharmacopsychiatry utilized data from approximately 1600 randomized controlled trials of 51 FGAs and 11 SGAs in the treatment of schizophrenia. ${ }^{31}$ Modest benefits were observed for the use of SGAs compared to FGAs for negative, cognitive, and depressive symptoms, and with a lower risk of tardive dyskinesia. These benefits were mainly attributed to the ability of SGAs to provide improvement in positive symptoms, equivalent to that of FGAs, with a lower risk of EPSE. There were no consistent differences between SGAs in terms of efficacy with the exception of clozapine, which was found to be more efficacious than other antipsychotics in people who had not responded to 1 or more other antipsychotics. Adequate trials of adequate doses of FGAs and SGAs were found to be key variables in optimizing effectiveness of antipsychotic agents. Substantial individual variability was observed in treatment response and adverse effects. SGAs offer the advantage of fewer acute extrapyramidal symptoms and less likelihood of tardive dyskinesia but produce greater metabolic side effects. Meta-analyses published subsequent to this summary statement and key trials on the use of clozapine are presented below.

\section{Clozapine monotherapy versus FGA agents Meta-analyses}

Leucht et al compared treatment outcomes between SGAs and FGAs in people with schizophrenia in general in a metaanalysis of 150 double-blind randomized studies including 21,533 participants. ${ }^{32}$ The meta-analysis by Essali et al also compared treatment outcomes between those taking FGAs 
versus SGAs and was largely based on the same data. ${ }^{33}$ Four SGA agents emerged as superior to FGA agents: clozapine, amisulpride, olanzapine, and risperidone. ${ }^{32}$ The majority of studies (121) were of 12 weeks' duration, 17 were of 6 months' duration, and 12 were longer than 12 months. It has been postulated that EPSE associated with FGAs may mimic the symptoms of schizophrenia and in early randomized controlled trials (RCTs) falsely suggested that SGAs are superior. ${ }^{34-36}$ In order to avoid this potential problem, only participants taking $\leq 12 \mathrm{mg} /$ day haloperidol (or $\leq 600 \mathrm{mg}$ /day chlorpromazine equivalents for low-potency FGAs) were included in this meta-analysis. Positive and Negative Symptom Scale (PANSS) and BPRS scores were used to assess overall efficacy and specific symptoms domains all of which were found to be more amenable to treatment with clozapine, olanzapine, amisulpride, or risperidone versus FGAs.

Treatment with clozapine produced medium effect sizes: overall symptoms -0.52 (95\% confidence intervals [CI]: -0.75 to $-0.29, P<0.0001$ ), positive symptoms -0.36 (CI: -0.56 to $-0.16, P<0.0001$ ), negative symptoms -0.27 (CI: -0.42 to $-0.13, P<0.0001$ ), depression -0.51 (CI: -0.87 to -0.14 , $P=0.006)$. Amisulpride and olanzapine produced similar improvements compared to FGAs: overall symptoms -0.31 (CI: -0.44 to $-0.19, P<0.0001)$ and -0.28 (CI: -0.38 to -0.18 , $P<0.0001$ ), respectively, positive symptoms -0.22 (CI: -0.37 to $-0.06, P=0.005$ ) and -0.15 (CI: -0.21 to $-0.09, P<0.0001$, negative symptoms -0.27 (CI: -0.40 to $-0.14, P<0.0001$ ) and -0.32 (CI: -0.47 to $-0.16, P<0.0001$ ), depression -0.37 (CI: -0.51 to $-0.24, P<0.0001$ ) and -0.27 (CI: -0.35 to -0.19 , $P<0.0001)$. The effect sizes associated with risperidone were small and the improvement observed on the depression subscale was not significant: overall symptoms -0.13 (CI: -0.22 to 0.05 , $P=0.002$ ), positive symptoms -0.13 (CI: -0.20 to $-0.05, P=0.001$ ), negative symptoms -0.13 (CI: -0.21 to $-0.06, P<0.0001$ ), depression -0.10 (CI: -0.23 to 0.03 , $P=0.145)$. Industry sponsorship, comparator dose, and prophylactic EPSE medication were assessed as moderator variables but did not yield any consistent effects. Leucht et al concluded that this reflects the fact that FGAs and SGAs are heterogeneous classes of compounds and argued that such categorization can lead to improper generalization and confusion. $^{32}$

\section{Randomized controlled trials}

Meltzer et al investigated the use of clozapine versus FGAs in treatment responsive participants during a 24-month study. ${ }^{37}$ Significant improvements in psychopathology, quality of life and global functioning were observed in both the clozapine $(n=40)$ and FGA group $(n=45)$ after taking a range of antipsychotic agents; most commonly haloperidol but also perphenazine, fluphenazine, loxapine, thioridazine, thiothixene, molindone, and amoxapine. While a similar improvement in psychopathology was observed, significantly more relapse/rehospitalization drop-outs occurred in those taking FGAs (19 relapse related hospitalizations in 10 participants versus 11 relapse related hospitalizations in 4 participants treated with clozapine). There were no differences in the occurrence of EPSEs between clozapine and the FGA groups; however, clozapine was associated with more weight gain.

In a 12-week double-blind trial, Krakowski et al randomly assigned participants with schizophrenia or schizoaffective disorder to receive clozapine $(n=33)$, olanzapine $(n=34)$, or haloperidol $(\mathrm{n}=33) \cdot{ }^{38}$ People with a history of nonresponse or intolerance to any of the 3 study medications were excluded. Aggression was assessed using the Modified Overt Aggression Scale (MOAS) and a cognitive task battery tested general executive function, visuospatial ability, psychomotor function, and visual and verbal memory. In the general cognitive index (GCI) no significant improvement was observed in the haloperidol or clozapine group while clozapine was the most efficient medication in reducing aggression. An important limitation was the concomitant, prophylactic use of benztropine $4 \mathrm{mg}$ /day for EPSE in the group taking haloperidol, which may increase anticholinergic cognitive impairment. Participants taking haloperidol showed no increase in body weight, blood lipids, or glucose. ${ }^{39}$

\section{Clozapine monotherapy versus other SGA agents \\ Meta-analyses}

The Cochrane Schizophrenia Group performed a metaanalysis in order to compare several commonly used SGA agents in terms of efficacy and tolerability in people with schizophrenia or schizophrenia-like psychoses. ${ }^{40}$ The primary outcome measure selected to assess this was change in total PANSS score, with positive and negative subscores as secondary outcomes. Outcomes were reported using weighted mean difference (WMD) in terms of PANSS scores and the dropout rate due to poor efficacy was included as a further outcome measure. Seventy-eight randomized, doubleblind studies were included for analysis of which 28 included treatment with clozapine.

The results relating to clozapine were different to those anticipated based on previous reports. No significant differences were found when comparing the total PANSS scores 
between clozapine and olanzapine $(\mathrm{N}=619)$, quetiapine $(\mathrm{N}=232)$, risperidone $(\mathrm{N}=466)$, or ziprasidone $(\mathrm{N}=146)$; however, clozapine was found to be significantly more efficacious than zotepine $(\mathrm{N}=59, \mathrm{WMD}=-6.0, P=0.002)$. The results for a decrease in positive symptoms reflected those found for overall symptoms while quetiapine was found to be more efficacious than clozapine on the negative symptom subscore $(\mathrm{N}=142, \mathrm{WMD}=2.2, P<0.001)$. Clozapine was favored over risperidone when comparing dropout rates due to poor efficacy $(\mathrm{N}=627$, relative risk $[\mathrm{RR}]=0.4095 \%$ confidence interval $[\mathrm{CI}]$ 0.23-0.70, $P=0.001)$. These unexpected results may be due to the low or very low doses of clozapine that were used in many of the studies included; several had an upper limit of $400 \mathrm{mg} /$ day and 5 used dosages under $210 \mathrm{mg} /$ day. In the pivotal studies that established clozapine's effectiveness, the average daily dose of clozapine was $600 \mathrm{mg} /$ day and $523 \mathrm{mg} /$ day. ${ }^{26,41}$ Furthermore the participants included in these trials may not have been as treatment refractory as those in other studies demonstrating clozapine's superiority over other SGA agents.

Substantial concerns about the side effects induced by SGA agents such as weight gain and metabolic syndrome may offset modest differences in their effectiveness. In a meta-analysis of head-to-head comparisons of the metabolic effects between SGA agents, Rummel-Kluge et al assessed weight gain and changes in cholesterol and glucose over 48 studies. $^{42}$ There were 3 main clusters in terms of these outcomes: olanzapine and clozapine produced the greatest elevation in weight, cholesterol and glucose (with no significant difference between the 2 agents) followed by quetiapine, risperidone, and sertindole with intermediate elevations. Aripiprazole and amisulpride showed lower elevations and ziprasidone the lowest. The authors noted that the dose of antipsychotic influenced some of the results in metaregressions; for example a high dose of olanzapine tended to produce a greater difference in the outcome measure in favor of the comparator drug. Another important caveat is that data on prior antipsychotic treatment for the participants in the selected studies were not available for analysis.

\section{Randomized controlled trials}

Phase II of the Clinical Antipsychotic Trials of Intervention Effectiveness (CATIE) recruited 99 participants who discontinued treatment with olanzapine, quetiapine, risperidone, or ziprasidone in phase I or IB of the trial primarily due to inadequate efficacy. ${ }^{43}$ Participants were randomized to blinded treatment with another newer SGA not previously received in the trial (olanzapine $n=19$, quetiapine $n=15$, or risperidone $n=16$ ) or open label treatment with clozapine $(n=49)$. At 3-month assessments, participants treated with clozapine experienced a greater reduction in PANSS total score $($ mean $=-11.7$, standard error $[\mathrm{SE}]=3.2)$ than participants treated with quetiapine (mean $=2.5, \mathrm{SE}=4.8$ ) or risperidone $($ mean $=4.1, \mathrm{SE}=1.9)$ but not olanzapine (mean $=-3.2, \mathrm{SE}=2.3$ ). Clozapine was significantly better only than quetiapine on the PANSS general psychopathology subscale $($ mean $=-4.7, \mathrm{SE}=1.5$ versus mean $=2.3, \mathrm{SE}=2.5$, $P=0.006$ ). Time to discontinuation for any reason was significantly longer for clozapine (median $=10.5$ months) than for risperidone (2.8 months) or quetiapine (median $=3.3$ months) but not olanzapine (median $=2.7$ months). Time to discontinuation is subject to bias in this phase of the study. Because treatment allocation was known to both clinicians and participants there may have been reluctance to discontinue clozapine, it being widely considered the best option for treatment-resistant schizophrenia. The data from this study support the conclusion that, for participants who prospectively failed to improve with an SGA, treatment with clozapine was more effective than switching to another SGA.

Phase III of CATIE allowed 270 participants who had discontinued antipsychotics in Phases I and II to select treatment from 9 antipsychotic regimens with the help of their study doctor. ${ }^{7}$ Approximately equal numbers of participants chose 7 of the 9 antipsychotics including clozapine (33-41 participants each agent). The study used a doubleblind design with the exception of those treated with clozapine, which was open label. The blinding of treatment with clozapine would have required additional monitoring of all treatment groups for clozapine specific safety issues, and in doing so may have affected the ecological validity of the other agents. All of the commonly used treatments were associated with substantial symptom improvement at 3 months and 6 months, with the exception of aripiprazole at 3 months and ziprasidone and quetiapine at 6 months. A total of 106 participants discontinued treatment; there were no significant differences in the proportions of participants who discontinued the commonly selected medicines (range 33\%-46\%). However, discontinuation due to lack of efficacy was lower for clozapine (5\%), risperidone, quetiapine, and ziprasidone $(0 \%-5 \%)$ than olanzapine, aripiprazole, and combination treatment $(13 \%-18 \%)$. Adverse effects were problematic in the group taking clozapine; the rates of adverse events classified as moderate or severe were highest for clozapine (35\%), quetiapine (45\%), and combination antipsychotic treat- 
ment (30\%). Clinically significant weight gain of at least $7 \%$ was common with clozapine (32\%), combination antipsychotic treatment (39\%), and olanzapine (23\%). All other SGA agents were associated with weight loss, in particular aripiprazole and ziprasidone, which produced the greatest monthly weight loss of $0.64 \mathrm{~kg}$ and $0.59 \mathrm{~kg}$, respectively; clozapine produced a gain of $0.59 \mathrm{~kg} /$ month.

Krakowski et al reported that olanzapine outperformed clozapine in terms of neurocognitive function in a study of 100 physically aggressive inpatients with schizophrenia or schizoaffective disorder. ${ }^{38}$ For metabolic parameters, participants taking olanzapine gained the most weight compared with clozapine or haloperidol, but clozapine was associated with the greatest increases in serum cholesterol, triglycerides and glucose. ${ }^{39}$ In the GCI olanzapine was found to be superior (improvement was approximately 0.5 standard deviations [SD]) to both clozapine and haloperidol; this was also associated with a decrease in aggression which was assessed using the MOAS. Rather than concluding that olanzapine has a procognitive effect it is perhaps more likely that olanzapine has less cognitive liability; clozapine has strong intrinsic anticholinergic activity compared with olanzapine. ${ }^{44}$ Nonetheless, treatment with clozapine markedly reduced aggression, suggesting that the antiaggressive effects of olanzapine may be mediated by different neuronal pathways.

It has been suggested that a decrease in serum cholesterol may result in aggression due to the subsequent decrease in brain serotonergic activity, given that cholesterol determines the availability of serotonin receptors and transporters. ${ }^{45}$ In a post-hoc analysis of the relationship between serum cholesterol levels and aggression in these groups, Krakowski and Czobor found a negative correlation at baseline. ${ }^{46}$ Based on changes in total cholesterol (TC) over the 12-week study period, the investigators used a Glimmix regression model to predict changes in aggression (Krakowski, pers comm). For those taking haloperidol it was predicted that a $141.9 \%$ increase in physical aggression was associated with a decrease of 1 SD unit in TC levels. Participants whose cholesterol increased by $1 \mathrm{SD}$ in the clozapine group were predicted to be $67.6 \%(P<0.001)$ less physically aggressive than those whose cholesterol did not change. It was then postulated that the antiaggressive effects of clozapine may have been further enhanced by an increase in cholesterol.

The UK Cost Utility of the Latest Antipsychotic Drugs in Schizophrenia Study 2 (CUtLASS 2) included 136 people with schizophrenia and related disorders whose medication was being changed due to suboptimal response to 2 or more previous antipsychotic agents. ${ }^{47}$ Participants were randomly allocated to receive clozapine or another SGA agent (risperidone, olanzapine, quetiapine, or amisulpride) selected by the treating clinician. The trial was rater-blind and outcome assessments were carried out for $87 \%$ of the participants at 12, 26, and 52 weeks following randomization. No significant advantage was observed for those taking clozapine compared with other SGA agents in the Quality of Life score (3.36 points, 95\% CI 0.46-7.71); however, a significant improvement was seen in the PANSS total score $(-4.93,95 \%$ $\mathrm{CI}-8.82$ to -1.05$)$. At 12 weeks the group taking clozapine reported that their mental health was significantly better than those taking other SGA agents. There were no significant differences between the treatment groups in the rate of adverse effects including weight gain.

Suicide has been identified as the leading cause of premature death among people with schizophrenia. ${ }^{48}$ The International Suicide Prevention Trial (InterSePT) assessed the risk for suicidal behavior in 980 participants with schizophrenia or schizoaffective disorder treated with clozapine compared to olanzapine over a 2 -year period. ${ }^{49} \mathrm{Par}-$ ticipants in this study, $26.8 \%$ of whom were refractory to previous treatment, were considered at high risk for suicide because of previous attempts or the presence of suicidal ideation. The study was conducted as an open-label trial with masked ratings. Suicidal behavior, defined as suicide attempts and hospitalizations to prevent suicide, was observed less frequently in those taking clozapine versus olanzapine (hazard ratio [HR] 0.76, 95\% CI 0.58-0.97). Worsening on the CGI-Suicide Severity or implicit worsening as demonstrated by occurrence of suicidal behavior was also less frequent in those taking clozapine (HR 0.78, 95\% CI 0.61-0.99). Fewer clozapine treated participants attempted suicide, required hospitalizations or rescue interventions to prevent suicide (34 versus 55, $P=0.03,82$ versus $107, P=0.05$ and 118 versus $155, P=0.01$, respectively). The need for concomitant antidepressants or anxiolytics/soporifics was also less frequent in those taking clozapine compared with olanzapine (221 versus 258 , $P=0.01$ and 301 versus $331, P=0.03$ ). Although the number of completed suicides was greater in the clozapine group ( 5 clozapine-treated participants versus 3 olanzapinetreated participants, $P=0.73$ ), this was not significant and the study was not powered to evaluate this as an endpoint. It was recognized by the investigators at the outset that the study would need to include 20,000 participants to detect 
a decreased relative risk for suicide deaths with clozapine therapy by $20 \%$.

In a randomized double-blind trial, Harvey et al compared the cognitive performance of 130 people with schizophrenia after 12 weeks of treatment with clozapine $(n=69)$ or ziprasidone $(\mathrm{n}=61) .{ }^{50}$ All participants were either resistant or intolerant to previous antipsychotic treatment. Clozapinetreated participants showed improvement on the Rey Auditory Verbal Learning Test (RAVLT; episodic memory) and the Stroop interference test (executive function) but not the Trail-Making Test (TMT; parts A and B; processing speed) compared with those taking ziprasidone. None of the individual items were observed to improve at 12 weeks between the treatment groups; however the composite score improved significantly in those taking ziprasidone compared with clozapine (effect size $\mathrm{D}=0.54, P=0.029$ ). One possible explanation for these results is that clozapine may interfere with the performance benefits of practice effects. Although it appears that ziprasidone is superior in reducing cognitive deficits in this short-term trial, clinical efficacy in terms of symptom control was not reported.

Davies et al compared clozapine to available SGA agents in a UK multi-center, rater-blind RCT in people with psychosis eligible for clozapine to assess cost-effectiveness. ${ }^{51}$ Over a 1-year period, it was found that clozapine was associated with higher quality-adjusted life years (QALYs) than other SGA agents, but at an additional cost. The probability that clozapine is cost-effective reached $50 \%$ if in order to gain 1 QALY the decision-makers were willing to pay $£ 33,000$. In other words, if the decision-makers were willing to pay less than $£ 33,000$ to gain 1 QALY, other SGA agents may be more cost-effective than clozapine. However, this trial was conducted with a relatively small number of participants ( $n=67$ clozapine; $n=69$ other SGA agents) and post-hoc calculations indicated that the power to detect significant differences in net money benefit was low $(50 \%$ if important differences in costs and QALYs were defined as $£ 1600$ and one-twentieth of a QALY, respectively). Furthermore, it may not be possible to extrapolate the results to longer-term clozapine treatment or to a population of primarily treatment-resistant people. The authors also noted that clozapine may be more cost-effective if fewer participants had clozapine initiated as an inpatient than in this RCT.

The present review found 2 RCTs comparing clozapine monotherapy with treatment with high-dose olanzapine ${ }^{52,53}$ and a further study examining treatment with ziprasidone with treatment-resistant participants. ${ }^{54}$ These studies will be discussed in detail below.

\section{Clozapine augmentation}

Despite proven efficacy in people with schizophrenia showing sub-optimal response to other antipsychotics, only $30 \%$ to $50 \%$ of people will experience clinically significant symptom improvement with clozapine treatment. ${ }^{29,30}$ One-third to two-thirds of people will continue to experience positive symptoms with adequate doses of clozapine or will be unable to reach adequate levels due to side effects that prevent further dose increases. ${ }^{30}$ Antipsychotic monotherapy is preferred over augmentation according to schizophrenia treatment algorithms; for people who do not respond to first-line antipsychotics, clozapine is recommended. Therefore clozapine augmentation strategies should be implemented only for those who experience insufficient response to clozapine monotherapy. An operational definition of nonresponse to clozapine or 'ultraresistant' schizophrenia is: BPRS improvement of $<20 \%$ despite a trial with clozapine for $\geq 8$ weeks and plasma levels $>350 \mu \mathrm{g} / \mathrm{L}$, no stable period of good social and/or occupational functioning for $\geq 5$ years, Global Assessment of Functioning (GAF) $\leq$ 40 , BPRS total score $\geq 45$, CGI score $\geq 4$, and a score of $\geq 4$ on 2 of 4 positive symptom items. ${ }^{55}$

\section{Meta-analyses}

\section{Augmentation with other antipsychotics}

The present review found 4 meta-analyses on the augmentation of clozapine treatment with another antipsychotic for people with an inadequate response to clozapine monotherapy ${ }^{56-59}$ These meta-analyses were based on essentially the same data, the largest of which was conducted by Barbui et al and arrived at similar conclusions with the exception of Correll et al. ${ }^{56,58}$

Barbui et al selected 21 studies to determine the efficacy of a second antipsychotic in combination with clozapine. ${ }^{56}$ The number of trials evaluating each augmentation agent was chlorpromazine $\mathrm{n}=1$, pipothiazine $\mathrm{n}=2$, amisulpride $\mathrm{n}=1$, sulpiride $\mathrm{n}=7$, and the remainder used risperidone $(\mathrm{n}=10)$. The mean length of follow up was 13.8 weeks $(\mathrm{SD}=19.6)$ and the trials were divided into either short-term studies of less than 10 weeks' duration or long-term studies. Clozapine combination strategies were favored in 14 open (nonblind), randomized studies in terms of effect size or standardized mean difference (SMD) from various outcome scales (SMD $=-0.80,95 \% \mathrm{CI}-1.14$ to -0.46 ). However, this trend was not apparent in 6 of the RCTs $(\mathrm{SMD}=-0.12$, $95 \%$ CI -0.57 to 0.32 ). Subgroup analysis by trial duration revealed a similar trend: the open studies favored clozapine combinations in both long- and short-term trials, the blinded 
studies showed no advantage for clozapine combinations of either duration.

Correll et al found antipsychotic combinations in general to be advantageous over monotherapy in a meta-analysis of 19 studies (1229 participants) in terms of all cause discontinuation ( $\mathrm{n}=1052$, RR $0.65,95 \%$ CI $0.54-0.78)$ and less study-specific inefficacy $(n=1202, \mathrm{RR} 0.76,95 \%$ CI $0.63-0.90) .{ }^{58}$ The mean trial duration was 12.1 weeks (range 4-52 weeks). The most commonly used antipsychotic was clozapine, though a variety of antipsychotic combinations were used. In terms of lack of efficacy as defined by each study, co-treatment including clozapine was superior to antipsychotic monotherapy $(\mathrm{n}=764$, RR $0.75,95 \%$ CI 0.61-0.93); however, the specific augmenting agents were not presented separately within the results. Meaningful results regarding specific psychopathology and adverse events could not be calculated due to insufficient data. Sensitivity analyses identified 5 efficacy moderators: clozapine combinations, concurrent polypharmacy initiation, Chinese trials, trial duration $>10$ weeks, and SGA-FGA combinations. Meta-regression of variables from sensitivity analyses identified 3 significant moderators associated with superior efficacy of antipsychotic combinations: similar doses in the mono- and polytherapy arm $(P=0.006$, coeff $=0.48), \mathrm{SGA}+\mathrm{FGA}$ combinations $(P=0.027$, coeff $=0.39$ ) and concurrent polypharmacy initiation $(P=0.050$, coeff $=0.35)$. The findings of this study differ from those of other meta-analyses of antipsychotic combination treatment and it is important to note that the positive results for antipsychotic combinations observed were primarily from Chinese studies not included in the other metaanalyses. A high degree of heterogeneity within the database and possible publication bias further obscured the significance of these findings.

Overall, it appears that the evidence considered for clozapine augmentation with another antipsychotic in these meta-analyses is weak and observed benefits are moderate at best. One consideration to take into account is that these reviews combined results of all antipsychotic augmentation irrespective of mechanism of action.

\section{Augmentation with anticonvulsants}

Dysfunctional glutamatergic neurotransmission is postulated to be an important component underlying the pathophysiology of schizophrenia. ${ }^{60}$ Lamotrigine is an anticonvulsant drug that inhibits excessive glutamate release in the brain by antagonism of sodium channels and increases gamma-zyric acid (GABA) release. It has been used as an augmenting agent on this basis. ${ }^{61,62}$ Tiihonen et al examined the advantages of combining clozapine with lamotrigine in 5 randomized placebo-controlled trials (161 participants) of 10 to 24 weeks' duration. ${ }^{63}$ On the primary outcome measure the total score for symptoms of psychosis, the clozapinelamotrigine combination was superior to the clozapineplacebo combination (SMD 0.57, 95\% CI 0.25-0.89; number needed to treat [NNT] 4, 95\% CI 3-6). The secondary outcome measures also favored this combination (SMD $0.34,95 \%$ CI $0.02-0.65$ for decreasing positive symptoms and SMD $0.43,95 \%$ CI $0.11-0.75$ for improving negative symptoms). The incidence of severe adverse effects or dropout rate did not differ between the treatment groups. No significant heterogeneity was observed in the meta-analysis. Importantly, this is the first evidence to date of efficacy for any pharmacological treatment in clozapine-resistant schizophrenia and it is noted by the authors that similar benefits may not be observed with lamotrigine and other antipsychotic agents apart from clozapine. The effect size for total score for symptoms of psychosis was 0.57 , suggesting beneficial effects for general symptoms which are known to be robust predictors of functional outcomes; however, scores were not available for all studies. ${ }^{64}$

\section{Augmentation with NMDA agonists}

Like anticonvulsants, the use of $N$-methyl-D-aspartate (NMDA) -enhancing agents is predicated on the glutamate hypothesis of schizophrenia, specifically NMDA receptor hypofunction. Antagonists of NMDA receptors such as phencyclidine and ketamine produce psychotic symptoms and neurocognitive deficits in human subjects and exacerbate psychotic symptoms in people with schizophrenia. ${ }^{65-67}$ Agonists at the obligatory NMDA-glycine binding site are glycine, $\mathrm{D}$-serine, and D-alanine and the partial agonist D-cycloserine, as opposed to agonists at the NMDA recognition site, which are excitotoxic. These agents, in addition to sarcosine which increases the availability of glycine in the synapse by inhibiting the glycine transporter-1 (GlyT-1), have been investigated as potential therapeutic agents for schizophrenia. Tsai and Lin performed a meta-analysis of 26 double-blind, placebo-controlled trials in approximately 800 people taking an NMDA agonist in addition to stable doses of antipsychotic medication for at least 4 weeks. ${ }^{68}$ Almost all studies used the PANSS to assess symptom severity. The pooled effect size of clinical efficacy of NMDA agonist augmentation compared with placebo for total psychopathology was 0.40 (95\% CI 0.22-0.58) and significant improvement was noted for depressive, negative, cognitive, positive, and general 
symptoms. Treatment with glycine, D-serine, and sarcosine was associated with improvement in multiple symptom domains while D-cycloserine was not. The concomitant antipsychotic used appeared to affect the efficacy of the NMDA-enhancing agent; those treated with risperidone or olanzapine improved, but those treated with clozapine did not. Gastrointestinal (GI) upset and nausea were noted more often in some glycine trials while other side effects were equivalent for NMDA-enhancing agents and placebo. Despite a moderate effect size, the efficacy of these agents may have been overstated due to limitations within the study. For instance, studies were included only if they provided "enough data to calculate the effect size" and a test for homogeneity revealed that there may have been systematic differences among the included studies. Another important caveat is that D-cycloserine, D-serine, D-alanine, and sarcosine are protected by US patents for which the study author is a patent holder.

\section{Randomized controlled trials} Augmentation with anticonvulsants

Topiramate is a GABAergic anticonvulsant drug indicated as add-on pharmacotherapy for adults and children with primary generalized tonic-clonic and partial-onset seizures. It has been used for people with schizophrenia to correct a postulated glutamate deregulation due to NMDA receptor hypofunction. Topiramate is thought to potentiate inhibitory GABAergic transmission (probably through a nonbenzodiazepine mechanism) and inhibit the activity of kainite on the AMPA/kainate receptor subtype. ${ }^{69-73}$

Two studies have examined the use of topiramate as an adjunct to treatment to clozapine with contrasting results. Afshar et al conducted a double-blind trial over 8 weeks with 32 people receiving clozapine treatment for at least 2 months. ${ }^{74}$ Participants were randomized to receive up to $300 \mathrm{mg} /$ day of topiramate $(\mathrm{n}=16)$ or placebo in addition to clozapine $(n=16)$. Total PANSS scores at baseline were similar between the groups, indicating a suboptimal response to clozapine monotherapy (topiramate group 96.87 \pm 21.98 ; placebo group $101.87 \pm 23.05, P=0.53$ ). Clinically significant improvement was defined as a $>20 \%$ decrease in total PANSS score and was observed in 8 participants $(50 \%)$ in the topiramate group and 2 in the placebo group (12.5\%; $P<0.05)$. The differences in the groups' total PANSS mean scores were reported at both 3 and 8 weeks and favored topiramate augmentation: $-11.18 \pm 8.72$ versus $-1.56 \pm 9.23, P=0.005$ and $-20.00 \pm 11.96$ versus $-1.31 \pm 11.13, P<0.001$, respectively. At 8 weeks a number of side effects were more prevalent in the topiramate group such as hypersalivation $(75.0 \%$ versus $34.7 \%$, $P \leq 0.05$ ) (although this was reported to be present in some participants prior to the study), psychomotor retardation (50.0\% versus $6.2 \%, P \leq 0.01)$, and paresthesia $(37.5 \%$ versus $6.2 \%, P \leq 0.05)$. Weight loss was also reported more commonly in the topiramate group $(37.5 \%$ versus $6.2 \%$, $P \leq 0.05)$. However, the authors reported that there were no differences observed in body mass index (BMI) between the groups or within each group over the trial period. None of the participants dropped out of the trial due to druginduced adverse effects. While the results of this small trial appear to favor topiramate augmentation, the follow-up period is relatively short. Furthermore, the investigators did not assess cognitive impairment, a well-documented, dosedependent adverse effect of topiramate that is particularly relevant to people with schizophrenia. ${ }^{75-78}$

The double-blind RCT by Muscatello et $\mathrm{al}^{79}$ was a methodologically robust 24 -week study that failed to replicate the benefits of topiramate add-on pharmacotherapy reported by Afshar et al. ${ }^{74}$ People receiving clozapine for at least 1 year, at a stable dose for at least 1 month, with a BPRS score of $\geq 25$ were eligible to participate. The clozapine dose remained unchanged throughout the study and participants noncompliant with all 10 study visits were excluded. No last observations were carried forward since this introduces assumptions which can under- or overestimate the effects of treatment. ${ }^{80}$ Participants did not receive any antidepressants or anticonvulsants for a period of 2 months prior to the study. A maximum dose of $200 \mathrm{mg}$ /day topiramate was added to clozapine treatment $(n=19$; placebo $n=24)$. No significant improvement in positive, negative, affective, or overall symptomatology from baseline to week 24 was observed. In the topiramate group a significant reduction was observed using the scale for the assessment of positive symptoms (SAPS) subscale for bizarre behavior (including clothing and appearance, aggressive behavior, stereotyped behavior and social, and sexual behavior). ${ }^{81}$ No significant effects on cognitive functioning were observed as measured by the Stroop test, verbal fluency, and the Wisconsin Card Sorting Test (WCST). No serious adverse events were reported; however, adjunctive topiramate was more frequently associated with asthenia, sedation, and paresthesia while constipation and hypersalivation were reported in the placebo group. There was no significant change in body weight from baseline to the end of the trial for the topiramate group. 
It is possible that this trial did not prove topiramate to be as useful for clinical symptomatology as the previous study because a lower dose of topiramate was used $(200 \mathrm{mg} /$ day versus $300 \mathrm{mg} /$ day). Yet this dose was chosen based on findings by Deutsch et $\mathrm{al}^{82}$ in order to avoid cognitive impairment which was not assessed by Afshar et al. ${ }^{74}$ Furthermore, the very small topiramate group $(n=19)$ means that only a large change in SAPS or WCST would produce a statistically significant difference. From these studies it appears that at doses low enough to preserve cognitive function, topiramate is of little benefit for clinical symptoms.

\section{Augmentation with cognitive enhancing agents}

Memantine is a weak, nonselective NMDA receptor antagonist approved for use in the treatment of moderate to severe Alzheimer's disease. De Lucena et al studied the effects of $20 \mathrm{mg}$ /day memantine combined with clozapine treatment for negative symptoms over 12 weeks. ${ }^{83}$ This double-blind trial was small (memantine $\mathrm{n}=10$, placebo $\mathrm{n}=11$ ) and consisted of those taking clozapine for at least 10 years for TRS. Significant improvements were seen at week 12 in the active treatment group for the total BPRS score (19.00 versus $43.18, P=0.001)$ and on the positive and negative symptom subscales (4.10 versus 9.18, $P=0.007$ and 6.10 versus 13.55 , $P=0.001)$. Those taking memantine also showed an 6.12-point (95\% CI 4.45-7.79) increase in mean score on the Mini-Mental State Examination (MMSE), although this is not the most sensitive measure of cognitive functioning. ${ }^{84}$ Simpson-Angus Scale (SAS) score and body weight were not significantly different between the groups. Based on results from animal studies, it has been postulated that memantine may improve cognitive function by upregulating the expression of brain-derived neurotrophic factor (BDNF) in humans. ${ }^{85}$ In this study, however, de Lucena did not detect an association between memantine treatment and increased serum BDNF levels, which have been highly correlated with cerebrospinal fluid BDNF levels. ${ }^{86}$ This may be due to the small sample size or clozapine treatment prior to randomization, which may also have increased serum BDNF levels. ${ }^{87}$

From this small trial, it appears that memantine may have beneficial effects in treatment-resistant people taking clozapine in particular; previous studies have not reported this effect in people taking atypical antipsychotics apart from clozapine. ${ }^{88}$ Other cognitive enhancing agents such as CX516 (an ampakine) and modafinil (a wakefulnesspromoting agent) have shown less promising results in recent randomized controlled trials. ${ }^{89,90}$ CX516 did not improve
PANSS scores after 4 weeks of co-administration with clozapine $(n=24)$, olanzapine $(n=18)$, or risperidone $(n=9)$ and was associated with fatigue, insomnia, and epigastric upset compared with placebo. ${ }^{90}$ In an 8-week trial, modafinil did not worsen psychosis in 35 people taking clozapine concurrently but also failed to reduce fatigue, negative symptoms, or cognitive deficits. ${ }^{89}$

\section{Augmentation with aripiprazole}

As a partial $\mathrm{D}_{2}$ agonist, aripiprazole's mechanism of action is distinct from that of other antipsychotics. It is a partial agonist at $5-\mathrm{HT}_{1 \mathrm{~A}}$ receptors, an agonist at $5-\mathrm{HT}_{2}$ receptors, and has been described as the prototype of a new generation of antipsychotic agents, the dopamine-serotonin system stabilizers. ${ }^{91}$ Partial agonism may be a beneficial property by allowing optimal neurotransmission, for instance, by acting as an antagonist in areas where there is an abundance of dopamine causing psychosis while acting as an agonist at receptor sites where low dopaminergic tone would produce adverse effects such as EPSE or hyperprolactinemia. ${ }^{92}$ Adverse effects associated with this drug such as somnolence, headache, light-headedness, and GI upset may be explained by its affinity for several other receptors including $\mathrm{D}_{3}, \mathrm{D}_{4}$, $5-\mathrm{HT}_{2 \mathrm{C}}, 5-\mathrm{HT}_{7}, \alpha_{1}$, and $\mathrm{H}_{1}$.

Millar et al studied aripiprazole or placebo in combination with clozapine in suboptimally controlled outpatients over a period of 16 weeks. ${ }^{93}$ Participants in this double-blind, randomized study were on a stable dose of clozapine for at least 3 months and had gained at least $2.5 \mathrm{~kg}$ since starting clozapine. At week 16, co-treatment with aripiprazole was associated with a significant decrease in mean weight compared with placebo (aripiprazole $2.53 \mathrm{~kg}$, placebo $0.018 \mathrm{~kg}$; $P<0.001$ ) and waist circumference (aripiprazole $-2.00 \mathrm{~cm}$, placebo $0 \mathrm{~cm} ; P<0.001)$. Both treatment groups showed similar improvement in the GAF. Improvements on the Epworth Sleepiness Scale and Fatigue Syndrome Inventory were observed in both groups; a significant difference in favor of aripiprazole was seen only in week 1 .

In an open-label extension of a 16 week double-blind placebo controlled trial (reviewed in the meta-analysis by Taylor and Smith 2009), ${ }^{59}$ Fleischhacker et al administered aripiprazole (5-15 mg/day) in combination with clozapine to all participants. ${ }^{94}$ For participants previously randomized to adjunctive placebo then treated with adjunctive aripiprazole for 12 weeks, the weight loss from the end of the double-blind phase was greater $(1.74 \mathrm{~kg}$ versus adjunctive aripiprazole $0.47 \mathrm{~kg}$ ). This finding suggests that while 
the weight loss was maintained in the initial aripiprazole group, this effect may plateau after a period of time. Clinically relevant weight loss from baseline was seen in $13 \%$ of those previously in the placebo group and in $21 \%$ of those taking aripiprazole for 28 weeks. Differences in PANSS scores were not significant between treatment groups in either phase of the study. The authors reported that symptom improvements were maintained; however, only the week 16 PANSS results were reported. Similarly, it was reported that participants who switched from placebo to aripiprazole at week 12 had reduced TC, low-density lipoprotein (LDL) cholesterol, and triglycerides but data illustrating this were not supplied.

Phase III of the CATIE study included only 2 participants receiving this combination of antipsychotics and therefore it was not meaningful to report these separately. However, the positive outcomes for weight loss in these randomized controlled trials correspond with findings in CATIE III; treatment with aripiprazole was associated with the most monthly weight loss $(0.64 \mathrm{~kg}){ }^{7}$ It appears from these trials that the addition of aripiprazole counteracts or at least decelerates the weight gain as a result of clozapine treatment without causing clinical deterioration or improvement.

\section{Options for clozapine-intolerant or clozapine-resistant people} Alternative antipsychotics

Two RCTs focused specifically on clozapine versus high dose olanzapine in TRS. ${ }^{53,95}$ Olanzapine is structurally similar to clozapine but has a different receptor affinity profile, being a weaker agonist for $\alpha_{1}$ and $\alpha_{2}$ receptors relative to its $\mathrm{D}_{2}, \mathrm{D}_{4}$, and $5 \mathrm{HT}_{2 \mathrm{~A}}$ antagonism. In a 6-month, doubleblind RCT Meltzer et al examined the efficacy and tolerability of high-dose olanzapine (target dose $25-45 \mathrm{mg} /$ day; mean dose $=34 \mathrm{mg} /$ day; $\mathrm{n}=19$ ) versus clozapine (target dose 300-900 mg/day; mean dose $=564 \mathrm{mg} /$ day; $\mathrm{n}=21$ ) in treatment-resistant participants with schizophrenia or schizoaffective disorder. ${ }^{53}$ Between 6 weeks and 6 months of treatment, significant and robust improvements were observed in both groups using multiple measures of psychopathology. The GAF significantly favored clozapine $(P<0.01)$; however, there were no other significant differences between each group. While it appears in this small trial that high-dose olanzapine was as effective as clozapine, significantly more weight gain in the olanzapine group may limit its use. At 6 months, the mean increase in BMI for those taking olanzapine was 2.2 versus 0.3 for those taking clozapine $(P=0.006)$.
Kumra et $\mathrm{al}^{52}$ concluded in a 12 -week controlled comparison of 39 adolescents with TRS that clozapine was superior to high-dose olanzapine (included in meta-analysis by Rummel-Kluge et al). ${ }^{42}$ In an open-label extension of this study, the authors investigated the metabolic side effects of these treatments at 24 weeks and the clinical response at 12 weeks of 10 of the 19 olanzapine-treated participants who were switched to clozapine due to nonresponse. ${ }^{95}$ Clinical response was defined as a decrease of at least $30 \%$ on the BPRS and a CGI-Improvement rating of 1 (very much improved) or 2 (much improved). On this basis, 7 of the 10 participants switched to clozapine were found to respond to clozapine. Metabolic side effects were similarly problematic in both treatment groups but direct comparisons between the groups were difficult to make due to the large proportion of participants switched to clozapine. It should also be noted that the mean weight of the participants at the beginning of this trial corresponded to a mean BMI percentile of 91.3 $(\mathrm{SD}=10.0)$, which may be accounted for by exposure to SGA agents prior to study entry.

With a much higher affinity for $5-\mathrm{HT}_{2}$ receptors than $\mathrm{D}_{2}$ receptors, ziprasidone has one of the highest serotonin/ dopamine binding ratios of the SGA group and a low affinity for $H_{1}$ and $\alpha_{1}$ receptors. Sacchetti et al investigated the use of ziprasidone compared to clozapine over an 18-week period in acutely unwell people (mean PANSS total score 107 ) with a history of multiple refractoriness to antipsychotics using a double-blind design. ${ }^{54}$ Decreases in the PANSS score were similar in each group; clozapine -24.5 $(95 \%$ CI -29.7 to -19.2$)$ and ziprasidone $-25.0(95 \%$ $\mathrm{CI}-30.2$ to -19.8$)$. Discontinuation rates due to adverse effects were similar however, ziprasidone offered the advantage of a more favorable metabolic profile (in terms of weight, fasting glucose, TC, LDL cholesterol, and triglycerides). Reductions in movement disorders assessed by the SAS and Abnormal Involuntary Movement Scale scores were also observed with ziprasidone but not clozapine. Clozapine-intolerant and clozapine-resistant participants were not distinguished from one another in this study which may have affected the results. The investigators also acknowledge that the mean dosage of clozapine (346 mg/day) was within the therapeutic range but lower than may be used in clinical practice.

\section{Nonpharmacological treatment}

A thorough appraisal of the value of nonpharmacological treatment options is beyond the scope of this review, however in the context of treatment resistance it is important to 
acknowledge the potential role of cognitive behavioral therapy (CBT) and electroconvulsive therapy (ECT).

\section{Cognitive behavioral therapy}

Recent studies have shown that CBT may be beneficial for those resistant to clozapine. Barretto et al compared CBT for psychosis $(n=12)$ to nonspecific social support also termed "befriending" (BF, $\mathrm{n}=9$ ) over 20 individualized therapy sessions over 3 weeks and at 6 months. ${ }^{96}$ The clozapine dose remained the same for all participants throughout the trial and the rater was blinded for the participants' intervention. At 6 months modest improvements were observed in the BPRS total score $(\mathrm{CBT}$ mean $=25.00, \mathrm{SD} 6.85$ versus BF mean $=19.00$, SD $8.38, P=0.0092)$, PANSS total score $(\mathrm{CBT}$ mean $=74.11, \mathrm{SD} 8.76$ versus $\mathrm{BF}$ mean $=66.54$ SD 13.95, $P=0.0447$ ), and PANSS general symptom subscale $(\mathrm{CBT}$ mean $=38.44, \mathrm{SD} 6.63$ versus $\mathrm{BF}$ mean $=33.45$ SD 7.27, $P=0.0147)$. Participants with residual negative symptoms such as conceptual disorganization, emotional withdrawal, and blunted affect were excluded from the study. Although this approach is rational, since such people may not be able to engage in and benefit from CBT, this limits the generalizability of the findings; many people with TRS have residual negative symptoms.

Turkington et al compared CBT $(n=31)$ and BF $(n=28)$ over a 5-year period in individuals with schizophrenia and persistent positive symptoms despite adequate trials of antipsychotic medication. ${ }^{97}$ Improvements were observed with CBT in overall symptoms severity $(\mathrm{NNT}=10.36,95 \%$ CI -10.21 to -10.51 ) and level of negative symptoms (NNT $=5.22,95 \%$ CI 5.06-5.37). While these results suggest that CBT may improve outcomes for participants, there was a significant break between the intervention which was completed at 9 months and follow-up at 18 months and 5 years. Intermediate follow-up assessments and booster sessions may have revealed greater benefits for CBT.

\section{Electroconvulsive therapy}

Matheson et al performed a systematic meta-review to determine the benefits and adverse outcomes associated with ECT and repetitive transcranial magnetic stimulation (rTMS) for people with schizophrenia. ${ }^{98}$ In contrast to ECT which produces global central nervous system excitation and generalized seizures, rTMS allows for targeted stimulation of superficial layers of the brain which may be effective for specific symptoms of schizophrenia. ${ }^{99}$ Furthermore, rTMS is subconvulsive and does not require an anesthetic or muscle relaxant. Five systematic reviews with meta-analysis were included in this meta-review (2 ECT, 3 rTMS) and graded in terms of the quality of evidence. High quality evidence suggested a short-term, small effect with ECT for the improvement of global symptoms in participants with or without concurrent antipsychotics $(\mathrm{RR}=0.76,95 \%$ CI $0.63-0.92) .{ }^{98,100}$ For rTMS, high quality evidence suggests a moderate to large decrease in auditory hallucinations $(\mathrm{D}=0.88,95 \% \mathrm{CI}$ 0.52-1.23). ${ }^{98,101}$ No evidence was found for long-term therapeutic or adverse effects of either treatment.

Lévy-Rueff et al conducted a retrospective chart review of 19 participants with schizophrenia or schizoaffective disorder nonresponsive or only partially responsive to pharmacological agents. ${ }^{102}$ In addition to antipsychotic medication, participants received maintenance ECT (M-ECT) beyond acute episodes of psychosis. Participants received an average of 47 sessions of bilateral M-ECT at 1- to 8-week intervals for a mean period of 43 months. Improvements in mood, delusions, anorexia, suicidal ideation, and anxiety were observed but symptom scores were not reported. With M-ECT the mean duration of yearly hospitalizations decreased by $80 \%$ within this cohort from 10.5 months (SD 17 months) in the year preceding M-ECT to 2.1 months (SD 2.04 months). The mean duration of each hospitalization decreased by $40 \%$, from 4.13 months (SD 4.0 months) prior to M-ECT to 2.53 months (SD 3.47). An improvement in daily functioning was also reported for most participants; 2 participants were discharged from full-time hospitalization and 1 returned to employment.

\section{Conclusion}

The results of the large trials CATIE and CUtLASS challenged the widely held belief that SGAs are superior to FGAs in treatment-responsive schizophrenia. One concept that remains unchanged is clozapine's superiority over both SGAs and FGAs in treatment-resistant schizophrenia; a finding reinforced by the second phase of each of these studies (and in the case of CATIE the third phase also) and the recent meta-analyses and RCTs presented in this review. In addition to people with treatment-resistant schizophrenia, studies suggest that clozapine may be useful for those at high risk of suicide or aggression. The adverse effects of clozapine are significant, ranging from acute events such as agranulocytosis to insidious weight gain and the onset of the metabolic syndrome. Many studies reported that clozapine treatment produced the greatest increase in BMI and/or body weight, closely followed by olanzapine.

The evidence supporting clozapine augmentation is weak and the benefits observed were moderate at best with the 
exception of lamotrigine. In the meta-analysis by Tiihonen et $\mathrm{a}^{63}$ lamotrigine produced significant improvements in the total PANSS or BPRS score and positive and negative symptom subscales. The use of the NMDA receptor antagonist memantine was supported by a recent RCT, which reported improvements in the MMSE, total BPRS, and positive and negative symptom subscales. Limited evidence suggests that NMDA agonists may produce clinical improvements in participants taking olanzapine or risperidone, but not clozapine, while the addition of topiramate to clozapine was of little benefit at doses low enough to preserve cognitive function. Clozapine augmentation with aripiprazole resulted in weight loss or at least halted further weight gain without causing clinical deterioration or improvement.

Recent RCTs suggest that high-dose olanzapine may be an important alternative for people intolerant or resistant to clozapine; evidence for the use of ziprasidone in these conditions is limited. CBT in addition to a nonclozapine antipsychotic for people not responding or intolerant to clozapine is supported by small trials. ECT (with or without concurrent antipsychotic medication) was found to produce small, short-term improvements in global functioning, while significant improvements specifically in auditory hallucinations were observed with rTMS. However, more studies are required to determine the long-term and adverse effects of these treatments.

In terms of clinical practice recommendations where there is a lack of evidence from RCTs to guide treatment, clinicians should review single case reports or case series, which are beyond the scope of this review. When implementing a treatment strategy for which there is limited evidence clinicians should ensure that the treatment trial is adequate with objective outcome measures, for example the PANSS. Larger trials with prospective data using clinically important outcomes measured by well-validated, approved instruments are needed to accurately compare the agents available for the treatment of schizophrenia. Future trials on clozapine augmentation strategies should aim to distinguish between augmenting agents rather than comparing the results of all antipsychotic augmentation irrespective of mechanism of action.

\section{Disclosure}

The authors declare no conflicts of interest.

\section{References}

1. Saha S, Chant D, Welham J, McGrath J. A systematic review of the prevalence of schizophrenia. PLoS Med. 2005;2(5):e141.
2. Marder SR. Initiatives to promote the discovery of drugs to improve cognitive function in severe mental illness. J Clin Psychiatry. 2006; 67(7):e03.

3. Gureje O, Herrman H, Harvey C, Morgan V, Jablensky A. The Australian national survey of psychotic disorders: Profile of psychosocial disability and its risk factors. Psychol Med. 2002;32(4):639-647.

4. Jablensky A, McGrath J, Herrman H, Castle D, Gureje O, Evans M, et al. Psychotic disorders in urban areas: An overview of the study on low prevalence disorders. Aust N Z J Psychiatry. 2000;34(2): 221-236.

5. Thornicroft G, Tansella M, Becker T, Knapp M, Leese M, Schene A, et al. The personal impact of schizophrenia in europe. Schizophr Res. 2004;69(2-3):125-132.

6. Wheeler A. Sociodemographic, functional and clinical correlates in outpatients with schizophrenia: Comparison with affective disorders. Aust N Z J Psychiatry. 2007;41(10):809-818.

7. Stroup TS, Lieberman JA, McEvoy JP, Davis SM, Swartz MS, Keefe RS, et al. Results of phase 3 of the catie schizophrenia trial. Schizophr Res. 2009;107(1):1-12.

8. Jones PB, Barnes TR, Davies L, Dunn G, Lloyd H, Hayhurst KP, et al. Randomized controlled trial of the effect on quality of life of second- vs first-generation antipsychotic drugs in schizophrenia: Cost utility of the latest antipsychotic drugs in schizophrenia study (cutlass 1). Arch Gen Psychiatry. 2006;63(10):1079-1087.

9. Lehman A LJ, Dixon L. APA practice guidelines: Schizophrenia. Am J Psychiatry. 2004;161(Suppl 2):1-56.

10. Carpenter W Jr, Heinrichs D, Wagman A. Deficit and nondeficit forms of schizophrenia: The concept. Am J Psychiatry. 1988;145(5): 578-583.

11. Erhart SM, Marder SR, Carpenter WT. Treatment of schizophrenia negative symptoms: Future prospects. Schizophr Bull. 32(2): 234-237.

12. Stahl SM, Buckley PF. Negative symptoms of schizophrenia: A problem that will not go away. Acta Psychiatr Scand. 2007;115(1):4-11.

13. Kirkpatrick B, Fenton WS, Carpenter WT, Marder SR. The nimhmatrics consensus statement on negative symptoms. Schizophr Bull. 2006;32(2):214-219.

14. Breier A, Schreiber JL, Dyer J, Pickar D. National institute of mental health longitudinal study of chronic schizophrenia: Prognosis and predictors of outcome. Arch Gen Psychiatry. 1991;48(3): 239-246.

15. Tandon R, Ribeiro SCM, DeQuardo JR, Goldman RS, Goodson J, Greden JF. Covariance of positive and negative symptoms during neuroleptic treatment in schizophrenia: A replication. Biol Psychiatry. 1993;34(7):495-497.

16. Harvey PD, Keefe RSE. Studies of cognitive change in patients with schizophrenia following novel antipsychotic treatment. Am J Psychiatry. 2001;158(2):176-184.

17. Mishara AL, Goldberg TE. A meta-analysis and critical review of the effects of conventional neuroleptic treatment on cognition in schizophrenia: Opening a closed book. Biol Psychiatry. 2004;55(10): 1013-1022.

18. Mortimer AM. Cognitive function in schizophrenia - do neuroleptics make a difference? Pharmacol Biochem Behav. 1997;56(4):789-795.

19. Bilder RM. Neurocognitive impairment in schizophrenia and how it affects treatment options. Can J Psychiatry. 1997;42(3):255-264.

20. Green MF, Braff DL. Translating the basic and clinical cognitive neuroscience of schizophrenia to drug development and clinical trials of antipsychotic medications. Biol Psychiatry. 2001;49(4): 374-384.

21. Tandon R, Nasrallah HA, Keshavan MS. Schizophrenia, "just the facts" 5. Treatment and prevention past, present, and future. Schizophr Res. 2010;122(1-3):1-23.

22. Goldberg TE, Goldman RS, Burdick KE, Malhotra AK, Lencz T, Patel RC, et al. Cognitive improvement after treatment with secondgeneration antipsychotic medications in first-episode schizophrenia: Is it a practice effect? Arch Gen Psychiatry. 2007;64(10):1115-1122. 
23. Davidson M, Galderisi S, Weiser M, Werbeloff N, Fleischhacker WW, Keefe RS, et al. Cognitive effects of antipsychotic drugs in first-episode schizophrenia and schizophreniform disorder: A randomized, open-label clinical trial (eufest). Am J Psychiatry. 2009;166(6):675-682.

24. Hill SK, Bishop JR, Palumbo D, Sweeney JA. Effect of secondgeneration antipsychotics on cognition: Current issues and future challenges. Expert Rev Neurother. 2009;10(1):43-57.

25. Keefe RSE, Bilder RM, Davis SM, Harvey PD, Palmer BW, Gold JM, et al. Neurocognitive effects of antipsychotic medications in patients with chronic schizophrenia in the catie trial. Arch Gen Psychiatry. 2007; 64(6):633-647.

26. Kane J, Honigfeld G, Singer J, Meltzer H. Clozapine for the treatmentresistant schizophrenic: A double-blind comparison with chlorpromazine. Arch Gen Psychiatry. 1988;45(9):789-796.

27. Kerwin RW, Bolonna A. Management of clozapine-resistant schizophrenia. Advances in Psychiatric Treatment. 2005;11(2):101-106.

28. Conley RR, Kelly DL. Management of treatment resistance in schizophrenia. Biol Psychiatry. 2001;50(11):898-911.

29. Buckley PF, Krowinski AC, Miller DD, Friedman L, Eaton Y, Tronetti M. Clinical and biochemical correlates of 'high-dose' clozapine therapy for treatment - refractory schizophrenia. Schizophr Res. 2001; 49(1-2):225-227.

30. Chakos M, Lieberman J, Hoffman E, Bradford D, Sheitman B. Effectiveness of second-generation antipsychotics in patients with treatmentresistant schizophrenia: A review and meta-analysis of randomized trials. Am J Psychiatry. 2001;158(4):518-526.

31. Tandon R, Belmaker RH, Gattaz WF, Lopez-Ibor JJ Jr, Okasha A, Singh B, et al. World psychiatric association pharmacopsychiatry section statement on comparative effectiveness of antipsychotics in the treatment of schizophrenia. Schizophr Res. 2008;100(1-3):20-38.

32. Leucht S, Corves C, Arbter D, Engel RR, Li C, Davis JM. Secondgeneration versus first-generation antipsychotic drugs for schizophrenia: A meta-analysis. Lancet. 2009;373(9657):31-41.

33. Essali A, Al-Haj Haasan N, Li C, Rathbone J. Clozapine versus typical neuroleptic medication for schizophrenia. Cochrane Database Syst Rev. 2009; 1:CD000059.

34. Geddes J, Freemantle N, Harrison P, Bebbington P. Atypical antipsychotics in the treatment of schizophrenia: Systematic overview and meta-regression analysis. BMJ. 2000;321(7273):1371-1376.

35. Leucht S, Wahlbeck K, Hamann J, Kissling W. New generation antipsychotics versus low-potency conventional antipsychotics: A systematic review and meta-analysis. Lancet. 2003;361(9369):1581-1589.

36. Rosenheck RA. Open forum: Effectiveness versus efficacy of secondgeneration antipsychotics: Haloperidol without anticholinergics as a comparator. Psychiatr Serv. 2005;56(1):85-92.

37. Meltzer HY, Bobo WV, Lee MA, Cola P, Jayathilake K. A randomized trial comparing clozapine and typical neuroleptic drugs in nontreatment-resistant schizophrenia. Psychiatry Res. 2010;177(3): 286-293

38. Krakowski MI, Czobor P, Nolan KA. Atypical antipsychotics, neurocognitive deficits, and aggression in schizophrenic patients. J Clin Psychopharmacol. 2008;28(5):485-493.

39. Krakowski M, Czobor P, Citrome L. Weight gain, metabolic parameters, and the impact of race in aggressive inpatients randomized to doubleblind clozapine, olanzapine or haloperidol. Schizophr Res. 2009; 110(1-3):95-102.

40. Leucht S, Komossa K, Rummel-Kluge C, Corves C, Hunger H, Schmid F, et al. A meta-analysis of head-to-head comparisons of second-generation antipsychotics in the treatment of schizophrenia. $\mathrm{Am}$ J Psychiatry. 2009;166(2):152-163.

41. Rosenheck R, Cramer J, Xu W, Thomas J, Henderson W, Frisman L, et al. A comparison of clozapine and haloperidol in hospitalized patients with refractory schizophrenia. N Engl J Med. 1997;337(12):809-815.

42. Rummel-Kluge C, Komossa K, Schwarz S, Hunger H, Schmid F, Lobos CA, et al. Head-to-head comparisons of metabolic side effects of second generation antipsychotics in the treatment of schizophrenia: A systematic review and meta-analysis. Schizophr Res. 2010;123:225-233.
43. McEvoy JP, Lieberman JA, Stroup TS, Davis SM, Meltzer HY, Rosenheck RA, et al. Effectiveness of clozapine versus olanzapine, quetiapine, and risperidone in patients with chronic schizophrenia who did not respond to prior atypical antipsychotic treatment. $\mathrm{Am} \mathrm{J}$ Psychiatry. 2006;163(4):600-610.

44. Chengappa KN, Pollock BG, Parepally H, Levine J, Kirshner MA, Brar JS, et al. Anticholinergic differences among patients receiving standard clinical doses of olanzapine or clozapine. JClin Psychopharmacol. 2000;20(3):311-316

45. Engelberg H. Low serum cholesterol and suicide. Lancet. 1992; 339(8795):727-729

46. Krakowski MI, Czobor P. A prospective longitudinal study of cholesterol and aggression in patients randomized to clozapine, olanzapine, and haloperidol. J Clin Psychopharmacol. 2010;30(2):198-200.

47. Lewis SW, Barnes TR, Davies L, Murray RM, Dunn G, Hayhurst KP, et al. Randomized controlled trial of effect of prescription of clozapine versus other second-generation antipsychotic drugs in resistant schizophrenia. Schizophr Bull. 2006;32(4):715-723.

48. Cohen LJ, Test MA, Brown RL. Suicide and schizophrenia: Data from a prospective community treatment study. Am J Psychiatry. 1990; 147(5):602-607.

49. Meltzer HY, Alphs L, Green AI, Altamura AC, Anand R, Bertoldi A, et al. Clozapine treatment for suicidality in schizophrenia: International suicide prevention trial (intersept). Arch Gen Psychiatry. 2003;60(1): 82-91.

50. Harvey PD, Sacchetti E, Galluzzo A, Romeo F, Gorini B, Bilder RM, et al. A randomized double-blind comparison of ziprasidone vs clozapine for cognition in patients with schizophrenia selected for resistance or intolerance to previous treatment. Schizophr Res. 2008; 105(1-3):138-143.

51. Davies LM, Barnes TR, Jones PB, Lewis S, Gaughran F, Hayhurst K, et al. A randomized controlled trial of the cost-utility of secondgeneration antipsychotics in people with psychosis and eligible for clozapine. Value Health. 2008;11(4):549-562.

52. Kumra S, Kranzler H, Gerbino-Rosen G, Kester HM, De Thomas C, Kafantaris V, et al. Clozapine and "high-dose" olanzapine in refractory early-onset schizophrenia: A 12-week randomized and double-blind comparison. Biol Psychiatry. 2008;63(5):524-529.

53. Meltzer HY, Bobo WV, Roy A, Jayathilake K, Chen Y, Ertugrul A, et al. A randomized, double-blind comparison of clozapine and highdose olanzapine in treatment-resistant patients with schizophrenia. J Clin Psychiatry. 2008;69(2):274-285.

54. Sacchetti E, Galluzzo A, Valsecchi P, Romeo F, Gorini B, Warrington L, et al. Ziprasidone vs clozapine in schizophrenia patients refractory to multiple antipsychotic treatments: The mozart study. Schizophr Res. 2009;113(1):112-21.

55. Mouaffak F, Tranulis C, Gourevitch R, Poirier MF, Douki S, Olie JP, et al. Augmentation strategies of clozapine with antipsychotics in the treatment of ultraresistant schizophrenia. Clin Neuropharmacol. 2006; 29:28-33.

56. Barbui C, Signoretti A, Mule S, Boso M, Cipriani A. Does the addition of a second antipsychotic drug improve clozapine treatment? Schizophr Bull. 2009;35(2):458-468.

57. Cipriani A, Boso M, Barbui C. Clozapine combined with different antipsychotic drugs for treatment resistant schizophrenia. Cochrane Database Syst Rev. 2009;3:CD006324.

58. Correll CU, Rummel-Kluge C, Corves C, Kane JM, Leucht S. Antipsychotic combinations vs monotherapy in schizophrenia: A meta-analysis of randomized controlled trials. Schizophr Bull. 2009;35(2): 443-457.

59. Taylor DM, Smith L. Augmentation of clozapine with a second antipsychotic - a meta-analysis of randomized, placebo-controlled studies. Acta Psychiatr Scand. 2009;119(6):419-425.

60. Goff DC, Coyle JT. The emerging role of glutamate in the pathophysiology and treatment of schizophrenia. Am J Psychiatry. 2001;158(9): $1367-1377$.

61. Cunningham MO, Jones RSG. The anticonvulsant, lamotrigine decreases spontaneous glutamate release but increases spontaneous gaba release in the rat entorhinal cortex in vitro. Neuropharmacology. 2000;39(11):2139-2146. 
62. Leach MJ, Baxter MG, Critchley MA. Neurochemical and behavioural aspects of lamotrigine. Epilepsia. 1991;32(Suppl 2):S4-S8.

63. Tiihonen J, Wahlbeck K, Kiviniemi V. The efficacy of lamotrigine in clozapine-resistant schizophrenia: A systematic review and meta-analysis. Schizophr Res. 2009;109(1-3):10-14.

64. Green MF. What are the functional consequences of neurocognitive deficits in schizophrenia? Am J Psychiatry. 1996;153(3):321-330.

65. Krystal JH, Karper LP, Seibyl JP, Freeman GK, Delaney R, Bremner JD, et al. Subanesthetic effects of the noncompetitive nmda antagonist, ketamine, in humans: Psychotomimetic, perceptual, cognitive, and neuroendocrine responses. Arch Gen Psychiatry. 1994;51(3): 199-214.

66. Malhotra AK, Pinals DA, Adler CM, Elman I, Clifton A, Pickar D, et al. Ketamine-induced exacerbation of psychotic symptoms and cognitive impairment in neuroleptic-free schizophrenics. Neuropsychopharmacology. 1997;17(3):141-150.

67. Lahti AC, Holcomb HH, Medoff DR, Tamminga CA. Ketamine activates psychosis and alters limbic blood flow in schizophrenia. Neuroreport. 1995;6(6):869-872.

68. Tsai GE, Lin PY. Strategies to enhance n-methyl-d-aspartate receptormediated neurotransmission in schizophrenia, a critical review and meta-analysis. Curr Pharm Des. 2010;16(5):522-537.

69. White HS, Brown SD, Woodhead JH, Skeen GA, Wolf HH. Topiramate enhances gaba-mediated chloride flux and gaba-evoked chloride currents in murine brain neurons and increases seizure threshold. Epilepsy Research. 1997;28(3):167-179.

70. Gibbs JW, Sombati S, DeLorenzo RJ, Coulter DA. Cellular actions of topiramate: Blockade of kainate-evoked inward currents in cultured hippocampal neurons. Epilepsia. 2000;41(Suppl 1):S10-S16.

71. Ängehagen M, Shank R, Hansson E, Rönnbäck L, Ben-Menachem E. Topiramate affects the ability of protein kinase to phosphorylate glutamate receptors activated by kainate Epilepsia. 2001;42 Supp1 7:S10.

72. Arnone D. Review of the use of topiramate for treatment of psychiatric disorders. Ann Gen Psychiatry. 2005;4(1):5.

73. Shank RP, Gardocki JF, Streeter AJ, Maryanoff BE. An overview of the preclinical aspects of topiramate: Pharmacology, pharmacokinetics, and mechanism of action. Epilepsia. 2000;41 Suppl 1:S3-S9.

74. Afshar H, Roohafza H, Mousavi G, Golchin S, Toghianifar N, Sadeghi M, et al. Topiramate add-on treatment in schizophrenia: A randomised, double-blind, placebo-controlled clinical trial. J Psychopharmacol. 2009;23(2):157-162.

75. Arif H, Buchsbaum R, Weintraub D, Pierro J, Resor SR Jr, Hirsch LJ. Patient-reported cognitive side effects of antiepileptic drugs: Predictors and comparison of all commonly used antiepileptic drugs. Epilepsy Behav. 2009;14(1):202-209.

76. Gilliam FG, Veloso F, Bomhof MA, Gazda SK, Biton V, Ter Bruggen JP, et al. A dose-comparison trial of topiramate as monotherapy in recently diagnosed partial epilepsy. Neurology. 2003;60(2):196-1202.

77. Lee S, Sziklas V, Andermann F, Farnham S, Risse G, Gustafson M, et al. The effects of adjunctive topiramate on cognitive function in patients with epilepsy. Epilepsia. 2003;44(3):339-347.

78. Thompson PJ, Baxendale SA, Duncan JS, Sander JW. Effects of topiramate on cognitive function. J Neurol Neurosurg Psychiatry. 2000; 69(5):636-641.

79. Muscatello MRA, Bruno A, Pandolfo G, Micò U, Bellinghieri PM, Scimeca G, et al. Topiramate augmentation of clozapine in schizophrenia: A double-blind, placebo-controlled study. J Psychopharmacology. 2010 Jul 8. [Epub ahead of print].

80. Streiner DL. The case of the missing data: Methods of dealing with dropouts and other research vagaries. Can J Psychiatry. 2002;47(1): 68-75.

81. Andreasen NC. The Scale for Assessment of Positive Symptoms (SAPS). Iowa City, IA; 1984. http://www.movementdisorders.org/UserFiles/file/ Long_SAPS_2000_publish(1).pdf.
82. Deutsch SI, Schwartz BL, Rosse RB, Mastropaolo J, Marvel CL, Drapalski AL. Adjuvant topiramate administration: A pharmacologic strategy for addressing nmda receptor hypofunction in schizophrenia. Clin Neuropharmacol. 2003;26(4):199-206.

83. De Lucena D, Fernandes BS, Berk M, Dodd S, Medeiros DW, Pedrini M, et al. Improvement of negative and positive symptoms in treatment-refractory schizophrenia: A double-blind, randomized, placebo-controlled trial with memantine as add-on therapy to clozapine. J Clin Psychiatry. 2009;70(10):1416-1423.

84. Lancu I, Olmer A. The minimental state examination - an up-to-date review. Harefuah. 2006;145(9):687-690, 701.

85. Meisner F, Scheller C, Kneitz S, Sopper S, Neuen-Jacob E, Riederer P, et al. Memantine upregulates bdnf and prevents dopamine deficits in siv-infected macaques: A novel pharmacological action of memantine. Neuropsychopharmacology. 2008;33(9):2228-2236.

86. Pan W, Banks WA, Fasold MB, Bluth J, Kastin AJ. Transport of brainderived neurotrophic factor across the blood-brain barrier. Neuropharmacology. 1998;37(12):1553-1561.

87. Gama CS, Andreazza AC, Kunz M, Berk M, Belmonte-de-Abreu PS, Kapczinski F. Serum levels of brain-derived neurotrophic factor in patients with schizophrenia and bipolar disorder. Neurosci Lett. 2007; 420(1):45-48.

88. Lieberman JA, Papadakis K, Csernansky J, Litman R, Volavka J, Jia XD, et al. A randomized, placebo-controlled study of memantine as adjunctive treatment in patients with schizophrenia. Neuropsychopharmacology. 2009;34(5):1322-1329.

89. Freudenreich O, Henderson DC, Macklin EA, Evins AE, Fan X, Cather C, et al. Modafinil for clozapine-treated schizophrenia patients: A double-blind, placebo-controlled pilot trial. J Clin Psychiatry. 2009;70(12):1674-1680.

90. Goff DC, Lamberti JS, Leon AC, Green MF, Miller AL, Patel J, et al. A placebo-controlled add-on trial of the ampakine, cx516, for cognitive deficits in schizophrenia. Neuropsychopharmacology. 2008;33(3): 465-472.

91. Tamminga CA, Carlsson A. Partial dopamine agonists and dopaminergic stabilizers, in the treatment of psychosis. Curr Drug Targets CNS Neurol Disord. 2002;1(2):141.

92. Rivas-Vasquez RA. Aripiprazole: A novel antipsychotic with dopamine stabilising properties. Prof Psychol Res Pr. 2003;34(1):108-111.

93. Millar H, Felter C, Landsberg W. The effects of aripiprazole in combination with clozapine: Patient functioning results from a double-blind, 16-week study in patients with schizophrenia (cn138-170). J Psychopharmacol. 2008;22(5):A17.

94. Fleischhacker WW, Heikkinen ME, Olie JP, Landsberg W, Dewaele P, McQuade RD, et al. Effects of adjunctive treatment with aripiprazole on body weight and clinical efficacy in schizophrenia patients treated with clozapine: A randomized, double-blind, placebo-controlled trial. Int J Neuropsychopharmcol. 2010;13(8):1115-1125.

95. Kumra S, Kranzler H, Gerbino-Rosen G, Kester HM, DeThomas C, Cullen K, et al. Clozapine versus "high-dose" olanzapine in refractory early-onset schizophrenia: An open-label extension study. J Child Adolesc Psychopharmacol. 2008;18(4):307-316.

96. Barretto EM, Kayo M, Avrichir BS, Sa AR, Camargo MG, Napolitano IC, et al. A preliminary controlled trial of cognitive behavioral therapy in clozapine-resistant schizophrenia. J Nerv Ment Dis. 2009;197(11): $865-868$.

97. Turkington D, Sensky T, Scott J, Barnes TRE, Nur U, Siddle R, et al. A randomized controlled trial of cognitive-behavior therapy for persistent symptoms in schizophrenia: A five-year follow-up. Schizophr Res. 2008;98(1-3):1-7.

98. Matheson SL, Green MJ, Loo C, Carr VJ. Quality assessment and comparison of evidence for electroconvulsive therapy and repetitive transcranial magnetic stimulation for schizophrenia: A systematic meta-review. Schizophr Res. 2010;118(1-3):201-210. 
99. Burt T, Lisanby SH, Sackeim HA. Neuropsychiatric applications of transcranial magnetic stimulation: A meta analysis. Int J Neuropsychopharmacol. 2002;5(1):73-103.

100. Tharyan P, Adams CE. Electroconvulsive therapy for schizophrenia. Cochrane Database Syst Rev. 2005;18(2):CD000076.

101. Aleman A, Sommer IE, Kahn RS. Efficacy of slow repetitive transcranial magnetic stimulation in the treatment of resistant auditory hallucinations in schizophrenia: A meta-analysis. $J$ Clin Psychiatry. 2007;68(3):416-421.
102. Lévy-Rueff M, Gourevitch R, Lôo H, Olié J-P, Amado I. Maintenance electroconvulsive therapy: An alternative treatment for refractory schizophrenia and schizoaffective disorders. Psychiatry Res. 2010 175(3):280-283.

\section{Publish your work in this journal}

Neuropsychiatric Disease and Treatment is an international, peer-reviewed journal of clinical therapeutics and pharmacology focusing on concise rapid reporting of clinical or pre-clinical studies on a range of neuropsychiatric and neurological disorders. This journal is indexed on PubMed Central, the 'PsycINFO' database and CAS, and is the official journal of The International Neuropsychiatric Association (INA). The manuscript management system is completely online and includes a very quick and fair peer-review system, which is all easy to use. Visit http://www.dovepress.com/testimonials.php to read real quotes from published authors.

Submit your manuscript here: http://www.dovepress.com/neuropsychiatric-disease-and-treatment-journal 